\title{
TOTALLY ENDOSCOPIC VENTRICULAR SEPTAL DEFECT CLOSED: HOW TO DO IT?
}

Do Anh Tien ${ }^{1,2}$, Le Ngoc Minh ${ }^{1,2}$, Tran Thuy Nguyen ${ }^{1,2 *}$, Nguyen Ba Phong ${ }^{1}$, Luu Phuong Linh ${ }^{1}$, Luong Thi Nhu Huyen ${ }^{1}$, Le Ngoc Thanh ${ }^{1,2}$

\section{ABTRACT}

Objectives: To describe the procedure of totally endoscopic closure of ventricular septal defect (VSD) without robotic assistance.

Methods: Totally endoscopic VSD closure was performed in 17 patiens (12 childrens and 5adults). The age was 8,5 year olds ( from 3 to 46), and the body weight was $31,2 \mathrm{~kg}$ (from 10 to 57 ). 16 patients were diagnosed with peri-membranous VSD and 1 infundibular VSD, in which 5 patients had right ventricular outflow tract stenosis. We used three $5 \mathrm{~mm}$-trocarts and one $12 \mathrm{~mm}$-trocart in the right chest. Using pperipheral cannulation for ccardiopulmonary bypass, superior vena caval occlusion, Chitwood aortic clamp, right atriotomy and closing VSD by totally endoscopy without a robotically assisted surgical system.

Results: There were no postoperative complications and deaths. cardiopulmonary bypass time and Aortic clamping time were 120 mins and 70 mins respectively. The mean mechanical ventilation time was 1.5 hours. Patients was hospitalized for 5.4 days and could resume normal daily activitive after 1 week.

Conclusions: Totally endoscopic closure of ventricular septal defect without robotic assistance is safe and feasible leaving only a small surgical scar with good aesthetics.
Keywords: Surgical Technique, Totally endoscopic closure, ventricular septal defect

\section{INTRODUCTION}

Ventricular septal defects (VSD) are the most common congenital intra-cardiac defects with high clinical importance [1]. The first successful closure of a VSD under direct vision was reported by Lillehei in 1955 . The procedure is normaly perfomed through a midline sternotomy incision and it is being widely applied all over the world [2]. Nowadays, with the developement of endoscopic surgery, VSD can be performed by endoscopic surgery $[2,3,4]$. Here we present a "How to do it?" article detailing the procedure.

\section{SURGICAL TECHNIQUE}

The patient lied in the left leaning position 30 degree, with endotracheal anesthesia. The anesthesis placed the central venous catheter through left internal jugular vein (IJV) and inserted a needle, which would be used for guidewire introduction for superior vena cava (SVC) cannulation - in the right IJV.

1 Department of Pediatric Cardiovascular Surgery, Cardiovascular Center E Hospital

2 University of Medicine and Pharmacy, National University Hanoi

*Corresponding author: Nguyen Tran Thuy

Email: drtranthuyvd@gmail.com

Address: E Hospital, 87-89 Tran Cung, Hanoi, Vietnam, 10000

Vietnam National University, 114 Xuan Thuy, Hanoi, Vietnam, 10000 


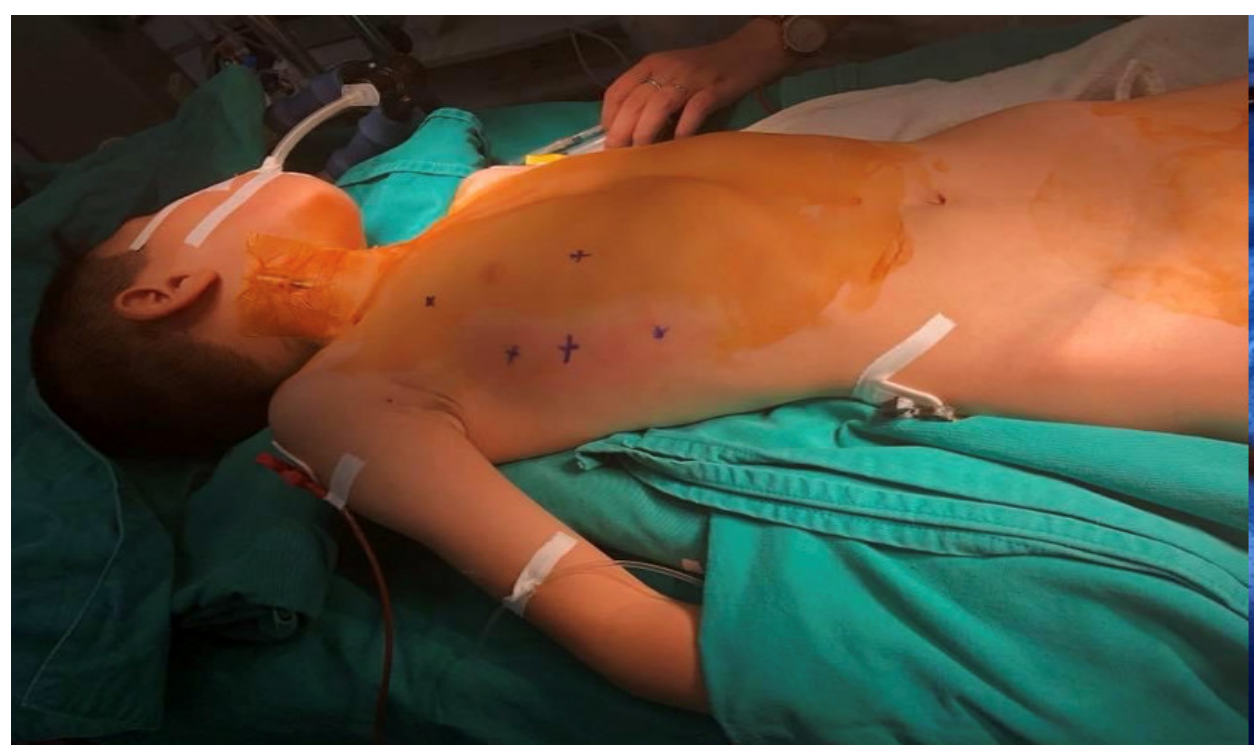

Figure 1: Prepare patient position

\section{Setting up peripheral cardiopulmonary bypass (CPB)}

We used a systemic doses of heparin $3 \mathrm{ml} / \mathrm{kg}$ body weight. We opened a mini incision on the right inguinal fold, revealing common femoral artery and femoral vein (for the inferior vena cavaIVC). SVC was placed through the right IJV with Seldinger technique. We tested the arterial line and the $\mathrm{CPB}$ was activated.

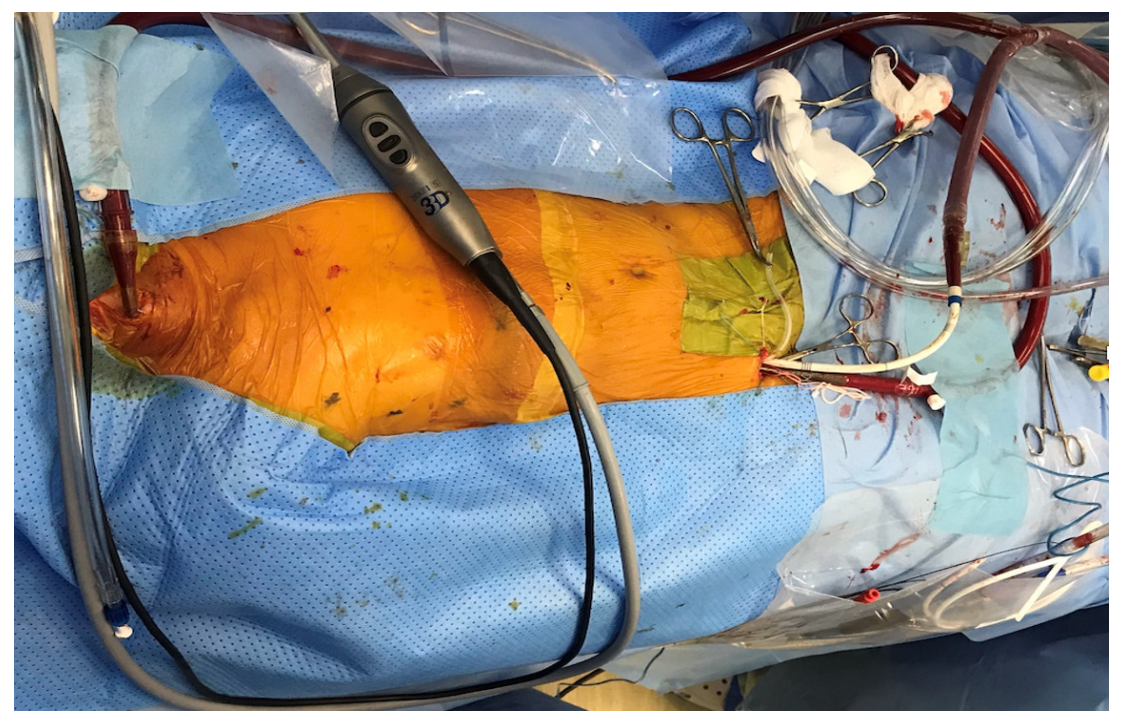

Figure 2: Peripheral cardiopulmonary bypass

Three trocars were placed in the previously marked positions. One $12 \mathrm{~mm}$-trocar was a main port at the sixth intercostal space in the midline collarbone. One $10 \mathrm{~mm}$-trocar was a camera port at the fifth intercostal space in the mid-axillary line. And the last one, $5 \mathrm{~mm}$-trocar was an assistance port at the fourth intercostal space in the anterior axillary line. 


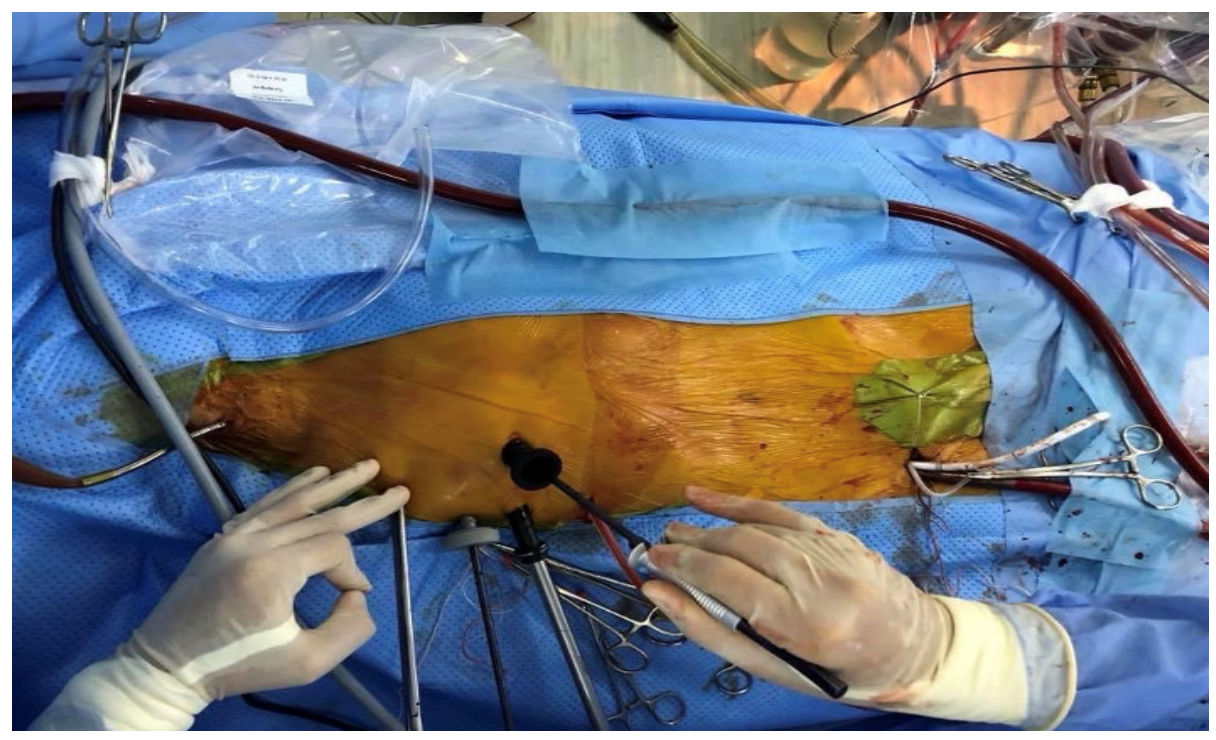

Figure 3: The port to surgery

After that, the pericardium was opened parallel and away $1.5 \mathrm{~cm}$ from the diaphragmatic nerve and suturing hangs to the chest wall. Then, we dissected SVC from the right pulmonary artery and snaring SVC. We inserted a cannulation into the aortic root. And we placed a needle into aortic root and using conventional hypothermic cardiopulmonary bypass with cold blood cardioplegia, using Chitwood clamp. The pleural and pericardia cavities were filled with $\mathrm{CO} 2$ and it was pumped at the rated of 11/min. Right atrial approach is chosen to close the VSD. We put the left vein through atrial septal. The ventricular septum is then explored through the tricuspid valve so that the defect could be seen and the anatomy assessed. We used technique patch closure with Interrupted Sutures or Continuous Sutures (pledget - supported)

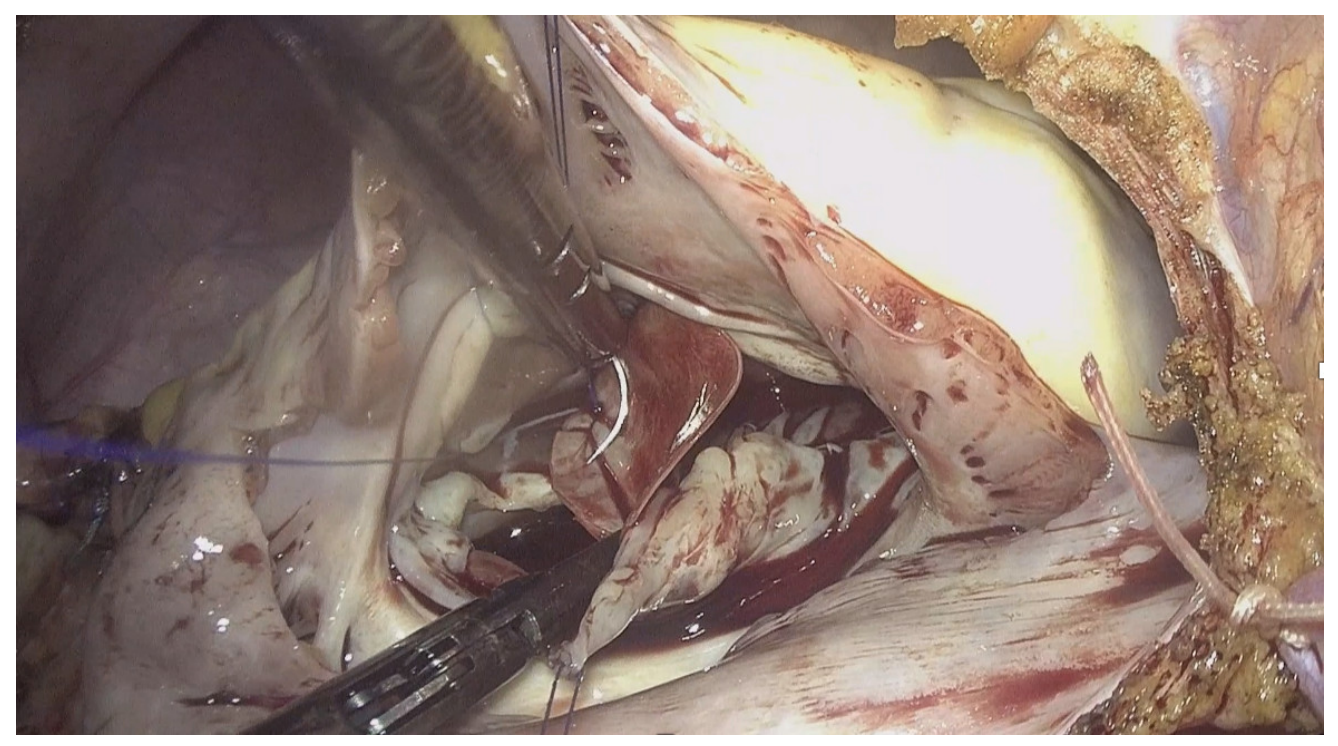

Figure 4: VSD exposure and patch close 
After finishing closure, we removed left vein and closed the heart opening lines. Removed Chitwood clamp and checked for any bleeding. We put one drainage of the right pleural cavity and closed all incisions.

Results: All patients had successfuly surgery by totally endoscopic with out robotic assistance. There were 16 perimembranous VSD and 1 infundibular VSD, 5 patients with right ventricular out flow obtruction.

Table 1: Clinical data

\begin{tabular}{lc}
\hline Variable & Value \\
\hline Patient & 17 \\
Adult & 5 \\
Children & 12 \\
Age (y) & $8,5(3-46)$ \\
Weight (kg) & $31,2(10-57)$ \\
Perimembrane & 16 \\
Infundibular & 1 \\
\hline
\end{tabular}

The mean cardiopulmonary bypass time and mean crossclamp time was 120 mins and 70 mins respectively. There were 13 patients with VSD patch close and 4 patients VSD directly close. 5 patients had RVOT enlargement concomitant by resect fibrous. The mean mechanical ventilation time was 1.5 hours. No mortality and no atrioventricular conduction block. The echocardiogram before discharge had no residual VSD with competence tricuspid valve and normal heart funtion. We had 1 patient with right femoral artey stenosis post operative who had been repair. Hospitalized for 5.4 days and could resume normal activitive after 1 week.

\section{Discussion}

The general trend of surgery in the world today is minimally invasive surgery. Minimally invasive cardiac surgery (MICS) is developed strongly in recent years which mainly applied to adult heart surgery with valvular heart disease $[5,6]$. However, for children, MICS is still a big problem for surgeons due to the small area of the surgical field, the lack of specialized tools.

For setting up peripheral cardiopulmonary bypass (CPB), we used common femoral artery and femoral vein. We have 1 patient with complications of femoral artery stenosis at the cannulation position. At first, we tried to place arterial cannula as big as possible. After facing this complication, we changed the strategy; we placed the cannula smaller than the diameter of the common femoral artery. We tested the arterial line pressure and the pressure of less than $250 \mathrm{mmHg}$ with full flow was acceptable. If the arterial line pressure was greater than $250 \mathrm{mmHg}$, we used one additional cannula (smaller than the previous one) in the opposite position to ensure enough blood perfusion. 
Superior and inferior vena cava cannula were placed through the right internal jugular vein and femoral vein under the guidance of trans-esophageal echocardiography.

We used conventional hypothermic cardiopulmonary bypass with cold blood cardioplegia (custodiol). We infused the solution downstream through the aortic root. After the infusion time was over, we removed the aortic root needle and performed enhanced sutures. Because we placed needle through the main port and if we did not pull out the needle, there would not be enough space to work. The problem was if the heart beat again during work, what should we do? With custodiol solution and deep hypothermia below 32 degree Celsius, the aortic clamping time was maximum 120 minutes. It was enough for us to close ventricular septal defect.

\section{Removing air}

We pumped $\mathrm{CO} 2$ continuously through a needle placed in the third intercostal space in the midline of clavicle. Before opening right atrial, we set up the patient into Tredenlenburg position. Because $\mathrm{CO} 2$ is heavier than others in the air, and it would be dissolved in the blood and it would not cause embolism complications [7,8]. To examination by trans-esophageal echocardiography, there was almost no air inside the heart.

Conclusions: Totally endoscopic closure of ventricular septal defect without robotic assistance is feasible with safe, a small surgical scar and hight aesthetics

\section{REFERENCE}

1. Penny DJ, Vick GW III (2011): Ventricular septal defect. Lancet;377: 1103-12.

2. Zeng -Shan et al (2012): Thoracoscopic closure of ventricular septal defect in young children: technical challenges and solutions. European Journal of Cardio-Thoracic Surgery, 42, 976-979

3. Changqing Gao, MD, Ming Yang, MD, Gang Wang, MD, Cangsong Xiao, MD, Jiali Wang, BS, MD, and Yue Zhao (2012): Totally endoscopic robotic ventricular septal defect repair in the adult. $J$ Thorac Cardiovasc Surg;144:1404-7

4. XingQ (2011): Transthoracic device closure of ven- tricular septal defects without cardiopulmonary bypass: experience in infants weighting less than $8 \mathrm{~kg}$. Eur $J$ Cardiothorac Surg ;40:591-7.

5. Mo X, Zuo W, Ma Z et al (2011). Hybrid procedure with cardiopulmonary bypass for muscular ventricular septal defects in children. Eur J Cardiothorac Surg ;40:1203-6.

6. Duc Hung Duong, Quoc Dat Pham (2019): Closure of subarterial ventricular septal defect with minimally invasive surgical technique: A

7. case report. International Journal of Surgery Case Reports 58, 142-144

8. Torracca L, Ismeno G, Alfieri O. Totally endoscopic computer-enhanced atrial septal defect closure in six patients. Ann Thorac Surg 2001;72:1354-7.

9. Yihu Tang, Yanhu Wu, Jinfu Zhu, Xiang Liu, Jinxin Zhou, Haobing Huang, Mingke Li, Yawei Dai, Xu Han (2018): Total endoscopic repair of atrial septal defect under on-pump beating heart. $J$ Thorac Dis 10(12):6557-6562 